\title{
COMMENTARY
}

\section{Magnesium sulfate for aneurysmal subarachnoid hemorrhage: the end of the road or more trials?}

\author{
Mervyn DI Vergouwen* \\ See related research by Wong et al., http://ccforum.com/content/15/1/R52
}

\begin{abstract}
Delayed cerebral ischemia (DCI) is a feared complication and an important cause of poor outcome after aneurysmal subarachnoid hemorrhage (SAH). In the current study, Wong and colleagues performed a systematic review and meta-analysis of randomized controlled trials that investigated the efficacy of magnesium sulfate in patients with aneurysmal SAH. Outcome measures were $\mathrm{DCl}$, cerebral infarction, and functional outcome 3 and 6 months after SAH. Magnesium sulfate decreased the rate of cerebral infarction, but not of DCl or poor functional outcome. Regarding outcome, a beneficial effect of magnesium sulfate on outcome can not be ruled out because of sample size limitations. Even if this meta-analysis had shown an effect on outcome, the question remains which treatment protocol should be applied in daily practice, since the administration of magnesium sulfate differed between most included studies. The present meta-analysis also underlines the importance of defining clinically relevant endpoints in SAH trials. Clinical deterioration due to $\mathrm{DCl}$ is more subject to inter-observer bias compared to cerebral infarction, which represents the ultimate outcome of the ischemic event. The Magnesium in Aneurysmal Subarachnoid Hemorrhage-II (MASH-II: ISRCTN68742385) phase III clinical trial nears completion. It aims to include 1,200 patients, and its results are urgently awaited.
\end{abstract}

Delayed cerebral ischemia (DCI) is a feared complication and an important cause of poor outcome after aneurysmal subarachnoid hemorrhage (SAH). Only limited progress has been made in the prevention of DCI. More than 20 years ago, the calcium antagonist nimodipine

*Correspondence: m.d.vergouwen@amc.uva.nl

University of Amsterdam, Academic Medical Center, Department of Experimental Vascular Medicine, Meibergdreef 9 (G1-145), 1105 AZ Amsterdam, the Netherlands was identified as a drug that decreases the risk of DCI. However, the effect of nimodipine is only modest. Many patients still develop this complication and suffer from its consequences. Therefore, new drugs are under investigation, to be used as adjunct therapy. Magnesium sulfate is a promising drug that is presently being investigated in randomized trials.

In the current study, Wong and colleagues performed a systematic review and meta-analysis update of randomized controlled trials that investigated the efficacy of magnesium sulfate in patients with aneurysmal SAH [1]. The present meta-analysis differs from previous metaanalyses in that it included the results of two recently published randomized trials [2,3]. Six trials were retrieved including 875 patients. Outcome measures were DCI, cerebral infarction, and functional outcome 3 and 6 months after SAH. The number of patients for the various meta-analyses ranged between 381 and 494 . The results showed that magnesium sulfate decreased the rate of cerebral infarction, but not of DCI or poor functional outcome.

Recently, it has been proposed that the main outcome measures of clinical trials and observational studies that investigate the complication of DCI should be cerebral infarction and functional outcome [4]. Arterial narrowing (vasospasm) is a radiographic finding associated with clinical deterioration due to DCI, cerebral infarction, and functional outcome. However, it is still under debate if these associations represent causal relationships. Vasospasm is often asymptomatic, and a reduction of vasospasm does not result in better functional outcomes [5]. Clinical deterioration due to DCI is less reliable than cerebral infarction, since it is more likely subject to interobserver bias. Cerebral infarction represents the ultimate outcome of the ischemic event, while clinical deterioration due to DCI is a diagnosis per exclusionem. Clinical deterioration after SAH can have multiple causes, such as infections, seizures, electrolyte disturbances, and hydrocephalus. The present meta-analysis used both DCI and cerebral infarction as outcome measures, and only found a beneficial effect on cerebral infarction. This effect on cerebral infarction did not translate to a beneficial effect 
on outcome, but as pointed out by the authors, an effect on outcome can not be ruled out because of the relatively low number of included patients.

Even if the present meta-analysis had shown a beneficial effect of magnesium sulfate on functional outcome, an important unanswered question would be which treatment protocol should be applied in daily practice. The administration of magnesium sulfate differed between most included studies. The concentration of magnesium sulfate was either 64 or $80 \mathrm{mmol} / \mathrm{day}$, with or without initial bolus, and with or without dosage adjustment according to serum magnesium levels. The Magnesium in Aneurysmal Subarachnoid Hemorrhage-II (MASH-II: ISRCTN68742385) phase III clinical trial will hopefully shed more light on the efficacy of magnesium sulfate in this group of patients. MASH-II aims to include 1,200 patients, which is based on a relative risk reduction of poor functional outcome of $22 \%$ (with alpha $=5 \%$ and a power of $80 \%$ ) [6]. In MASH-II, magnesium sulfate $64 \mathrm{mmol} /$ day or placebo is started within 4 days after $\mathrm{SAH}$ and continued until 20 days after the hemorrhage. This trial nears completion and its results are to be expected soon.

\section{Abbreviations}

$\mathrm{DCl}$, delayed cerebral ischemia; MASH-II, Magnesium in Aneurysmal

Subarachnoid Hemorrhage-II; SAH, subarachnoid hemorrhage.

Competing interests

The author declares that he has no competing interests.

\section{Acknowledgements}

Mervyn DI Vergouwen is financially supported by a grant from the Netherlands Thrombosis Foundation, The Netherlands (2010-4).
Published: 25 March 2011

References

1. Wong GKC, Boet R, Poon WS, Chan MTV, Gin T, Ng SCP, Zee BCY: Intravenous magnesium sulphate for aneurysmal subarachnoid hemorrhage: an updated systemic review and meta-analysis. Crit Care 2011, 15:R52.

2. Wong GK, Poon WS, Boet R, Chan MT, Gin T, Ng SC, Zee B, IMASH investigators: Intravenous magnesium sulphate after aneurysmal subarachnoid hemorrhage: a multi-center phase III study. Stroke 2010 41:921-926.

3. Westermaier T, Stetter C, Vince GH, Pham M, Tejon JP, Eriskat J, Kunze E, Matthies C, Ernestus RI, Solymosi L, Roosen K: Prophylactic intravenous magnesium sulfate for treatment of aneurysmal subarachnoid hemorrhage: a randomized placebo-controlled, clinical study. Crit Care Med 2010, 38:1284-1290.

4. Vergouwen MD, Vermeulen M, van Gijn J, Rinkel GJ, Wijdicks EF, Muizelaar JP, Mendelow AD, Juvela S, Yonas H, Terbrugge KG, Macdonald RL, Diringer MN, Broderick JP, Dreier JP, Roos YB: Definition of delayed cerebral ischemia after aneurysmal subarachnoid hemorrhage as an outcome event in clinical trials and observational studies: proposal of a multidisciplinary research group. Stroke 2010, 41:2391-2395.

5. Etminan $N$, Vergouwen MD, llodigwe $D$, Macdonald RL: Effect of pharmaceutical treatment on vasospasm, delayed cerebral ischemia, and clinical outcome in patients with aneurysmal subarachnoid hemorrhage: a systematic review and meta-analysis. J Cereb Blood Flow Metab 2011 [Epub ahead of print].

6. Dorhout Mees SM; MASH-II study group: Magnesium in aneurysmal subarachnoid hemorrhage (MASH II) phase III clinical trial MASH-II study group. Int J Stroke 2008, 3:63-65.

doi:10.1186/cc10055

Cite this article as: Vergouwen MDI: Magnesium sulfate for aneurysmal subarachnoid hemorrhage: the end of the road or more trials? Critical Care 2011, 15:140. 